\title{
Numerical Simulation about Trapping Two Particles in Microfluidic Dielectrophoretic Chip
}

\author{
Liguo Chen ${ }^{1}$, Shaoqian $\mathrm{Li}^{2}$, Haihang $\mathrm{Cui}^{3}, \mathrm{Xu} \mathrm{Zheng}^{4}$ \\ ${ }^{1}$ Robotics and Microsystems Center, Soochow University, Suzhou, China \\ ${ }^{2}$ Shanghai Engineering Center for Microsatellites, Shanghai, China \\ ${ }^{3}$ School of Environment and Municipal Engineering, Xi'an University of Architecture and Technology, Xi' an, China \\ ${ }^{4}$ Institute of Mechanics, LNM, Chinese Academy of Science, Beijing, China
}

Corresponding-lishaoqian@,hit.edu.cn

\begin{abstract}
A kind of microfluidic chip based on dielectrophoresis (DEP) was designed for the purpose of trapping two particles with different sizes. In order to reach the purpose, the shape of electrode and the shape of the flow channel was designed, the electric field and the flow field of the chip was simulated. At last, the trajectory of the particles was obtained. The results show that the chip can complete pairing two particles with different sizes.
\end{abstract}

Keywords - Microfluidic Chip, DEP, Particle Trapping.

\section{INTRODUCTION}

Cell fusion is a very important technology in the field of life sciences, the technology not only provides a powerful means for the theoretical research in different fields, such as nuclear mass relations [1], genetic complementation [2], gene mapping [3], gene regulation [4], membrane protein dynamics [5], disease prevention [6], but also is widely used in genetics [ 7 ], immunology[ 8 ], developmental biology. With the development of cell fusion technology, it is believed that animals, plants and microbial cell fusion techniques will become increasingly significant in practical applications.

A very important step of Cell fusion process is to pair and capture two particles, and then use biological or chemical methods to make them fuse together.

Pairing and capturing of equal size or almost equal size particles has made great progress. Yang Jun, from Chongqing University, put forward cell electrofusion chip based on the polyimide substrate [9], and achieved queuing and fusion of animal cells by this chip. This chip can integrate a lot of microelectrode on a chip (integration can be as high as 10000 electrodes $/ \mathrm{cm} 2$ ) to achieve high throughput in order to obtain a sufficient number of fusion cells for post- training. Skelley, from the Massachusetts Institute of Technology, who published an article on the Nature Methods, developed a cell electrofusion chip based on PDMS (Polydimethylsiloxane), The cell capture device comprises thousands of PDMS cell traps densely arrayed within a flow-through channel. Using this device, they paired different cell types, including fibroblasts, mouse embryonic stem cells and myeloma cells, achieving the pairing efficiency up to $70 \%$. The device is compatible with both chemical and electrical fusion protocols $[10]$.
Pairing particles with vary sizes has still not been studied. Combination of the demand to capture a pair of particles in the cell fusion and the advantage of dielectrophoresis in particle trapping, this subject carried out the research of particle pairing and particle capturing based on microfluidic chip, which has the ability to capture pairs of particles with vary sizes.

\section{THEORY}

The time average DEP force of particle is given by

$$
\left\langle F_{D E P}\right\rangle=2 \pi \varepsilon_{0} \varepsilon_{m} R^{3} \operatorname{Re}\left[C M^{*}\right] \nabla|E|^{2}
$$

where $\mathrm{R}$ is the radius of the particle, $\varepsilon_{0}$ is permittivity of vacuum, $\varepsilon_{m}$ is permittivity of medium, $\nabla|E|^{2}$ is the gradient of RMS electrical field, $C M^{*}$ is the Clausius-Mossotti factor. $\operatorname{Re}\left[C M^{*}\right]$ is the real part of $C M^{*}$,

$$
\begin{aligned}
C M^{*} & =\left(\frac{\varepsilon_{p}^{*}-\varepsilon_{m}{ }^{*}}{\varepsilon_{p}{ }^{*}+2 \varepsilon_{m}{ }^{*}}\right) \\
\varepsilon_{p}^{*} & =\varepsilon_{p}-j \frac{\sigma_{p}}{\omega} \\
\varepsilon_{m}{ }^{*} & =\varepsilon_{m}-j \frac{\sigma_{m}}{\omega}
\end{aligned}
$$

Where $\varepsilon_{p}$ is the permittivity of particle, $\sigma_{p}$ is the conductivity of particle, $\sigma_{m}$ is the conductivity of fluid medium, $\omega$ is angular frequency of electrical field, $j=\sqrt{-1}$.

When the spherical particles move in unbounded viscous fluid slowly, it is resisted by the fluid. The force is known as Stokes force, and its expression of $\overline{F_{s t}}$ is given by

$$
\overrightarrow{F_{s t}}=6 \pi \mu R(\vec{u}-\vec{v})
$$

where $\mu$ is the viscosity of fluid medium, $\mathrm{R}$ is radius of 
particle, $\vec{u}$ is velocity of flow, $\vec{v}$ is the velocity of particle.

In addition to the DEP force and Stokes force, the particles withstand gravity, buoyancy, wall friction, molecular forces (Van Der Waals forces), Saffman force (the lift force caused by the velocity gradient) and Magnus force (the force after the collision with the wall), as well as the interaction between particles. Approximately counteract due to very small density difference of the polystyrene microspheres (about $\left.1200 \mathrm{~kg} / \mathrm{m}^{3}\right)$ and the water $\left(1000 \mathrm{~kg} / \mathrm{m}^{3}\right)$ close to the influence of gravity and buoyancy. Because the mass of the microspheres is very little, the acceleration is very large, so it can be regarded as a mass-free particle processing and ignores the acceleration process. According to the literature, the impact of the remaining force will be ignored. Therefore, applying equation (1) and (3) into $\vec{F}_{D E P}+\vec{F}_{s t}=0$, we can get the velocity of the particles under the DEP force and Stokes force, equals to

$$
\vec{u}_{p}=\frac{\varepsilon_{0} \varepsilon_{m} r^{2} \operatorname{Re}\left[C M^{*}\right] \nabla|E|^{2}}{3 \mu}+\vec{u}
$$

\section{Design Of Micro Fludic ChIP}

Microfluidic chip model is shown in Fig. 1. The chip consists of three parts, substrate, cover and micro-flow channel. A layer of $100 \mathrm{~nm}$ thick microelectrode is located on the substrate. A number of U-shaped obstacles in the micro flow channel are designed for particle capture, which are made of SU-8.

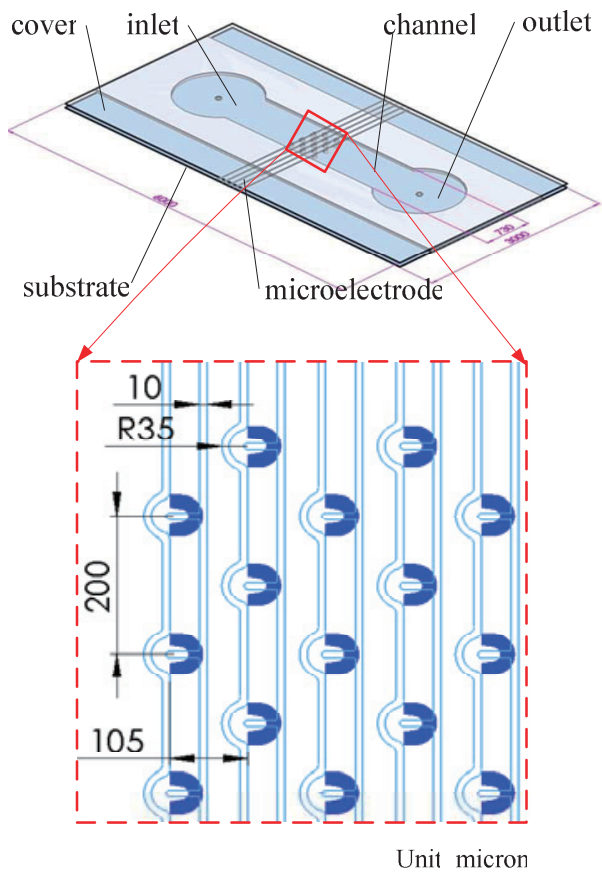

Fig. 1. Scheme of microfluidic chip to capture a pair of particles with different sizes

Principle to capture a pair of particles is shown in Fig. 2. The processes to capture a pair of particles are:
1) In Fig. 2a), particle A (diameter $=20 \mu \mathrm{m})$ in the suspension are driven by the inflow from the left inlet. Because a stream line flows through the gap of the Ushaped obstacles, particle A will be driven along into the gap. Considering that the gap $(15 \mu \mathrm{m})$ from the substrate to the bottom of the U-shaped obstacle is not sufficient to allow particle A pass through it, particle A will be captured in the U-shaped obstacle gap.

2) Due to the presence of particle A, blocking the flow of suspensions, the flow lines will not be through the gap, therefore, when the second particle through here it will bypass the U-shaped gap, as shown in Fag. 2b). This ensures that a U-shaped obstacle can only capture one particle A.

3) Electrify the microelectrode which is plated on the substrate, at the tip of the electrode electric field strength is most large, adjusting the suspension conductivity, so that particle $\mathrm{A}$ is driven by pDEP force, as shown in Fag. 2c), the particle A will be firmly adsorbed on the tip of the electrode.

4) The particles B through the inlet flow into the chip, because the existence of with pDEP force, particle B will be adsorbed to the electrode tip and close together with the particle A. By regulating the size of the electrode voltage and suspension flow rate, the tip of the electrode can only capture one particle B.

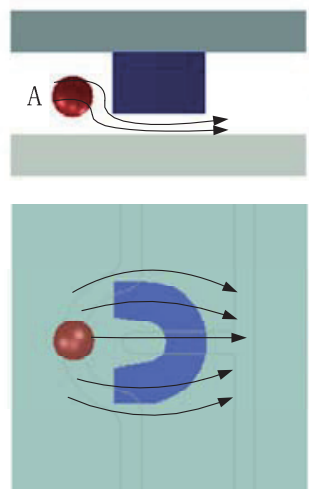

a)

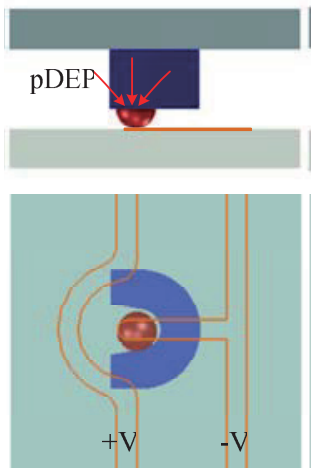

c)
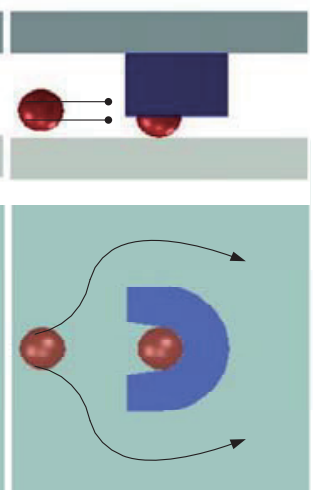

b)

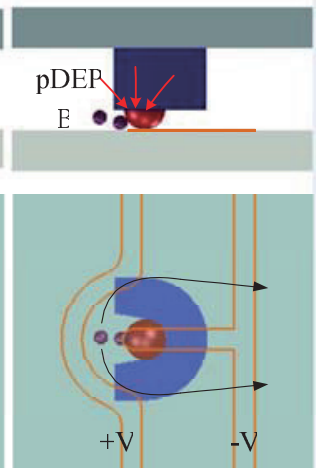

d)

Fig 2. Principle to capture two particles with different sizes in turn 
To achieve the purpose of mass capture, just manufacture the module array on the substrate. A proper module distance need to be set, in order to ensure capture efficiency and reduce the probability of the chip is blocked.

\section{SiMULATION OF THE CHIP}

Take into account both the accuracy and efficiency of simulation, geometric module of Microfluidic chip need to be simplified. The smallest unit of the chip is used for simulation analysis, and its three-dimensional model in Comsol Multiphysics software is shown in Fig. 3. Establishment of a Cartesian coordinate system is shown in Fig. 3. The parameters of each part is shown in Table 1.

TABLE 1

PHYSICAL PROPERTIES USED IN SIMULATION

\begin{tabular}{|c|c|c|c|c|c|c|c|}
\hline \multicolumn{4}{|c|}{ Relative Permittivity } & \multirow{3}{*}{$\begin{array}{c}\text { Vacuum Permittivity } \\
(F / m)\end{array}$} & \multicolumn{2}{|c|}{$\operatorname{Density}\left(\mathrm{Kg} / \mathrm{m}^{3}\right)$} & \multirow{2}{*}{$\begin{array}{c}\text { Viscosity } \\
(P a \cdot s)\end{array}$} \\
\hline Particle & Fluid & $S U-8$ & Substrate & & Particle & Fluid & \\
\hline$\varepsilon_{p}$ & $\varepsilon_{m}$ & $\varepsilon_{U}$ & $\varepsilon_{s}$ & & $\rho_{p}$ & $\rho_{m}$ & $v_{m}$ \\
\hline 2.55 & 78 & 3.0 & 3.5 & $8.854 \times 10^{-12}$ & $1.2 \times 10^{3}$ & $10^{-3}$ & $10^{-3}$ \\
\hline
\end{tabular}

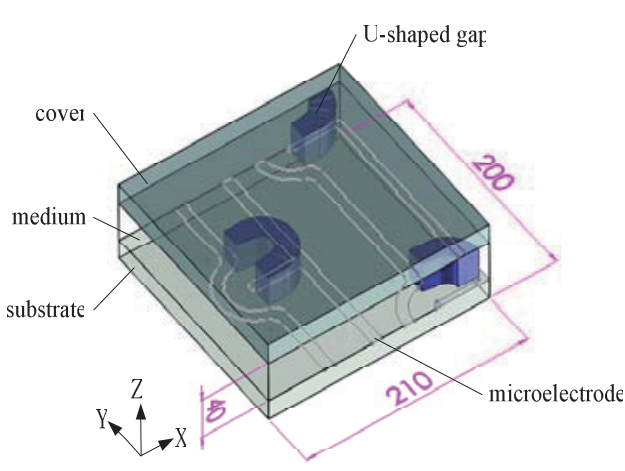

Fig. 3. A trapping unit to realize the function to capture a pair of particles

\section{A. Design and Simulation of Microelectrode}

Equation (1) shows that the shape of the electrode determines the magnitude of $E \cdot \nabla E$, which also determines the strength of the DEP force. In order to achieve high enough electric field in the specified location, the electrode structure is shown in Fig. 4a). The width of electrode is $10 \mu \mathrm{m}$. Set $1 \mathrm{~V}$ initial voltage on the electrode. On surfaces that parallel with plane yz and xz, periodic boundary conditions are adopt, corresponding equations are:

$$
V_{s r c}=V_{d s t}
$$

The entire chip meet Laplace equation:

$$
\begin{aligned}
& \nabla \cdot D=0 \\
& E=-\nabla V \\
& D=\varepsilon_{0} \varepsilon_{r} E
\end{aligned}
$$

The distribution of electric field on the electrode plane is shown in Fig. 4b), maximum electric field intensity $\left(3.9 \times 10^{5} \mathrm{~V} / \mathrm{m}\right)$ is obtained at the electrode tip. From Fig. 4c), there are relatively high electric field strength in the other two places. In order to ensure that obtained by the microelectrode electric field strength at the tip of microelectrode is significantly higher than other locations, a larger radius filleting the electrode need to be used. Using the radius $(\mathrm{R}=10 \mu \mathrm{m})$ of the fillet can reduce the electric field strength of the corner as shown in Fig. 4d).

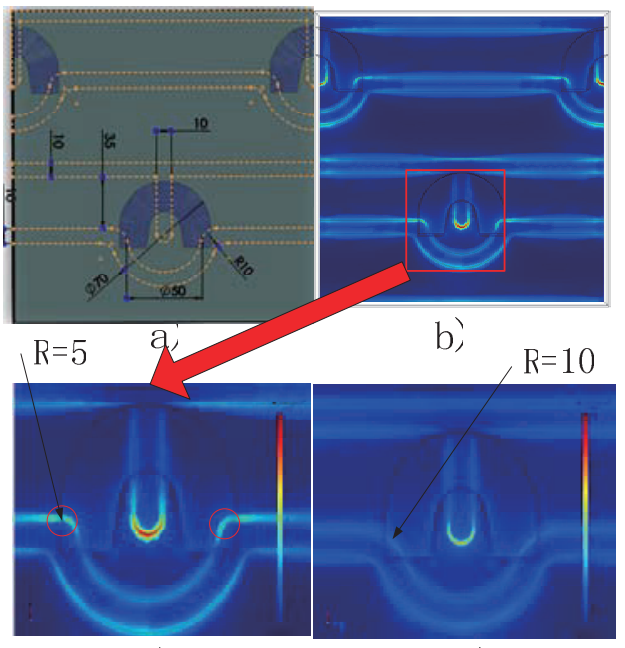

C)

d'

Fig. 4. Design of the fillet microelectrode and the resultant electric field

\section{B. Design and Simulation of the Channel}

To ensure that just one particle is captured, a reasonable shape of the U-shaped barrier is necessary to design. Designed U-shaped obstacle is shown in Fig. 5a), the channel height is $40 \mu \mathrm{m}, \mathrm{U}$-shaped obstacle height is $30 \mu \mathrm{m}$. Periodic boundary condition is set in upstream side and downstream side surface of the channel, pressure difference between upstream side and downstream side is $1 \mathrm{~Pa}$, the corresponding boundary equations are:

$$
\begin{gathered}
u_{\text {source }}=u_{\text {dest }} \\
p_{\text {sourse }}=p_{\text {dest }} \\
p_{\text {src }}-p_{d s t}=1 P a
\end{gathered}
$$

At the right and left surfaces of the channel, symmetry boundary conditions are used. The boundary equations are:

$$
\begin{gathered}
\vec{u} \cdot \vec{n}=0 \\
\vec{K}-(\vec{K} \cdot \vec{n}) \vec{n}=0 \\
\vec{K}=\left[\mu\left(\nabla \vec{u}+(\nabla \vec{u})^{T}\right)\right] \vec{n}
\end{gathered}
$$


Reynolds number of the entire flow field is far less than 1 , so it is laminar flow, $\mathrm{N}-\mathrm{S}$ equations is simplified:

$$
\begin{gathered}
\rho(\vec{u} \cdot \nabla) \vec{u}=\nabla \cdot\left[-\rho \vec{I}+\mu\left(\nabla \vec{u}+(\nabla \vec{u})^{T}\right)\right]+\vec{F} \\
\rho \nabla \cdot \vec{u}=0
\end{gathered}
$$

The simulation results are shown in Fig. 5b), it can be seen that the flow line through the gap of the U-shaped obstacle.

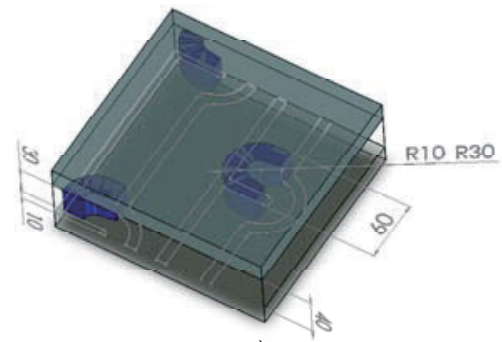

a)

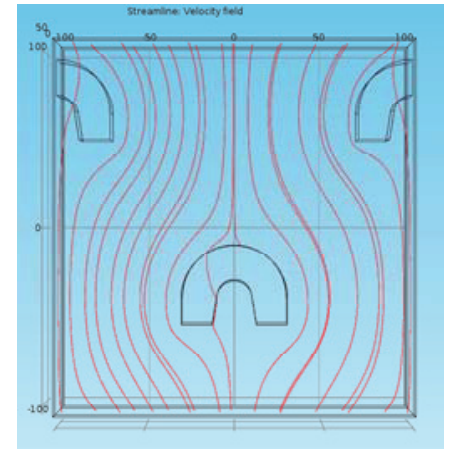

k)

Fig. 5. simulation of flow field inside the micro channel with U-shape structure

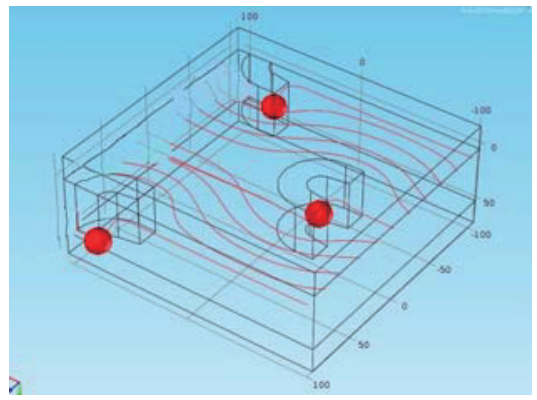

Fig. 6. Single particle can be trapped by flow field

Put particles in the flow field, the trajectories of particles in the chip can be drawn, as shown in Fig. 6. It can be see that each three U-shaped column captures one particle, when the particles is stuck in the U-shaped mouth, the followed particle will not be captured at the same location any more.

\section{CONCLUSION}

In this work, the purpose that pairing and capturing a pair of particles with different sizes is studied. Combined the advantage of DEP force that it can manipulate particles at particular position, a kind of microfluidic chip based on pDEP is designed to finish the function by two steps. The numerical simulation proved that:

(1)The first particle can be captured by the U-shaped structure without the help of pDEP. Due to the presence of the first particle, the following particles will pass by;

(2)In the second step, the microelectrode is charged. pDEP force is obtained on the particles and attracted them to the tip of the microelectrode. By regulating the size of the electrode voltage and suspension flow rate, the tip of the electrode can only capture one particle B. So the chip completes its function of capturing a pair of particles.

A series of experiments to prove the chip's function is doing now.

\section{ACKNOWLEDGMENT}

This work is supported by National Natural Science Foundation of China (the Grant Number 21005058, 61106110, 51105263, 51105262), the National Project (the Grant Number 2012AA040404 - 2011ZX04013-012), the Funds of the State Key Lab of Fluid Power Transmission and Control (the Grant Number GZKF-201028), State Key Laboratory of Transducer Technology(the Grant Number SKT1004) and Opening fund of State Key Laboratory of Nonlinear Mechanics. And the authors would like to think all members of the NSFC and the State Key Lay.

\section{REFERENCES}

[1] Hayashi T, Tanaka J. "Immunogenicity and therapeutic effacacy of Dendrentic tum or hybrid cells generated by electro fusion". Clinical Immunology, , vol. 104, no. 1, pp.14-20, 2002

[2] Yamagishi H, Landgren M, Forsberg J, et al. "Product ion of asymmetric hybrids between Arabidopsis thaliana and Brassicanapus utilizing an efficient protoplast culture system". Theoretical and Applied Genetics, vol. 104, no. 6-7, pp.959-964, 2002.

[3] Schmalzing, D., Belenky, A., Novotny, M.A., et al. "Microchip Electrophoresis: A Method for High-Speed SNP Detection", Nucleic Acid Research, in press.

[4] Tian H, Jaquins-Gerstl A, Munro N, et al. "Single-strand conformation polymorphism analysis by capillary and microchip electrophoresis: a fast, simple method for detection of common mutations in BRCA1 and BRCA2", Genomics, pp.63:65, 2000.

[5] Peter Nagy, Laszlo Matyus, Attila Jenei. "Cell fusion experiments reveal distinctly different association characteristics of cell surf ace receptors". Journal of Cell Science, vol. 114, no.22, pp.4063-4071, 2001.

[6] Robert H, Broyles R. "Use of somatic cell fusion to reprogram globin genes". Seminars in Cell and Development al Biology, vol. 10, no.3, pp.259-265, 1999.

[7] Khandurina J, McKnight T E, Jacobson S C, et al. "Integrated system for rapid PCR-based DNA analysis in microfluidic devices", Anal Chem, , vol. 72, no. 13, pp.2995-3000, 2000.

[8] Abad-Villar E M, Tanyanyiwa J, Fernandez-Abedul M T, et al. "Detection of human immunoglobulin in microchip and conventional capillary electrophoresis with contactless conductivity measurements", Anal Chem,vol.76, no.5, pp.1282-1288, 2004.

[9] Hu Ning, Yang Jun, Zheng Xiaolin, et al. "Polyimide Membrane Based Flexible Cell-electrofusion Chip". Chinese Journal of Analytical Chemistry, vol.37, no.28, pp.1247-1250, 2009.

[10] Skelley AM, Kirak O, Suh H, et al. "Microfluidic control of cell pairing and fusion". Nature Methods, vol.6, no.2, pp.147-152, 2009. 